\title{
Accelerating the Pace of Newborn Screening Research to Advance Disease Understanding and Improve Health Outcomes:
}

\section{Key Efforts of the Newborn Screening Translational Research Network (NBSTRN)}

Amy Brower, PhD; Kee Chan, PhD, MPH; Jennifer Taylor, PhD; Ross Wiebenga; Galata Tona, MS; Yekaterina Unnikumaran, MS; LaStephanie Barnes

American College of Medical Genetics and Genomics (ACMG)

\begin{abstract}
Each year in the United States, neonatal screening leads to approximately 1 in 220 newborns being identified with a condition that requires treatment, and in some cases, life-saving interventions. Research that discovers new technologies to screen, diagnose, and treat diseases helps to expand the number of conditions that are candidates for nationwide screening. The National Institute of Child Health and Human Development (NICHD) Hunter Kelly Newborn Screening Research Program funds research to advance newborn screening (NBS). A key effort is the Newborn Screening Translational Research Network (NBSTRN). For fourteen years, the American College of Medical Genetics and Genomics (ACMG) has developed and coordinated the activities of the NBSTRN. This article provides an overview of the NBSTRN.
\end{abstract}

Newborn screening (NBS) is a multi-component system that involves education, neonatal screening and diagnosis, followed treatment, and in some cases, life-long management. In the United States (U.S.), approximately 1 in 220 newborns is diagnosed with a condition through NBS. State-based public health departments, like the Delaware Newborn Screening Program (NSP), play a key role in organizing and conducting screening and referral to care. While each state determines which conditions to screen, a federal advisory committee, the Advisory Committee of Heritable Disorders in Newborns and Children (ACHDNC), has established a Recommended Uniform Screening Panel (RUSP) of thirty-five core and twenty-six secondary conditions. ACHDNC has also established a nomination and evidence review system, available to the NBS community of researchers, healthcare professionals, families, and advocacy groups to expand NBS to conditions that are not yet part of screening. NBS research leads to discoveries and pilots of conditions that are candidates for screening, and eventual, RUSP nomination.

NBS began in the 1960s with the discovery that a biomarker for a metabolic disorder could be detected in a newborn's blood. Elevation of this biomarker led to the diagnosis of phenylketonuria (PKU) and timely interventions that prevented the development of intellectual disability. The enormous benefit of pre-symptomatic diagnosis and treatment of PKU, made possible by NBS, has fostered over six decades of collaborative efforts between research, public health, and clinical care to expand the number of screened conditions. Leading research efforts is the National Institute of Child Health and Human Development (NICHD), one of the twentyseven institutes and centers at the National Institutes of Health (NIH). NICHD operates the Hunter Kelly Newborn Screening Research Program to fund research to advance NBS. The goals of the Hunter Kelly Newborn Screening Research Program are to identify, develop and test promising new screening technologies; increase the specificity of newborn screening; expand the number of conditions for which screening tests are available; and/or develop experimental treatments and disease management strategies for additional newborn conditions, and other 
genetic, metabolic, hormonal and or functional conditions that can be detected through newborn screening for which treatment is not yet available. A key effort is the Newborn Screening Translational Research Network (NBSTRN). For fourteen years, the American College of Medical Genetics and Genomics (ACMG) has developed and coordinated the activities of the NBSTRN.

NBSTRN develops data tools, resources, and expertise to facilitate and support ground-breaking research to accelerate understanding of genetic disease to increase the number of screened conditions and foster collaborations with clinicians, families, and health professionals to understand and maximize health outcomes. NBSTRN tools are designed to help plan research studies, facilitate efforts, accelerate discoveries, and foster collaborations with key stakeholders and partners. A description of NBSTRN tools highlights the different facets of NBS research and the important role public health plays in advancing disease understanding and improving outcomes for all newborns.

\section{Longitudinal Follow-Up with the LPDR}

Because newborn screening aims to improve health outcomes by identifying and treating affected newborns, NBSTRN created a data tool to facilitate the longitudinal collection of health information on newborns diagnosed with a condition through NBS. The Longitudinal Pediatric Data Resource (LPDR) captures, stores, analyzes, visualizes, and shares genomic and phenotypic data over the lifespan of NBS identified newborns to facilitate understanding of genetic disease and to assess the impact of early identification and treatment.

\section{State-based Newborn Screening Programs with the NBS-VR}

In the United States, there are 53 newborn screening (NBS) programs located in 50 states, two territories, and the District of Columbia (D.C.). Although there are federal recommendations, each NBS program establishes its policies and procedures, including: which conditions to screen, whether and how to store residual dried blood spots (DBS), whether to obtain consent from parents for the use of DBS, and whether to conduct long-term follow-up of diagnosed cases. NBSTRN created the NBS Virtual Repository of States, Subjects \& Samples (NBS-VR) to provide national and state-level views of these policies and procedures. The NBS-VR facilitates collaboration with NBS programs. The NBS-VR consists of an interactive map of the United States and an interactive table to download data.

\section{NBS Conditions with the NBS-CR}

Currently, newborns in the U.S. are screened for 81 disorders, 61 by the ACHDNC, and 20 conditions not on the RUSP are screened by at least one state. An additional 35 conditions have been identified as candidates for pilots to determine whether screening is feasible and beneficial. In addition to the screened conditions, thousands of rare disorders may be candidates for NBS. The Newborn Screening Conditions Resource (NBS-CR) provides a centralized resource of facts and statistics on both screened and candidate conditions. The NBS-CR is designed to be an interactive resource for researchers, clinicians, parents, and families to learn more about these disorders and links to National Library of Medicine (NLM) resources, including the National Center for Biotechnology Information (NCBI). NCBI provides access to biomedical and genomic information and maintains MedGen, NCBI's portal, to information about human 
disorders and phenotypes having a genetic component. Use the filter tool to sort conditions by nomination status and ACHDNC category.

\section{Ethical Considerations in NBS with ELSI Advantage}

The ELSI Advantage is a tool developed by the NBSTRN to aid the newborn screening research community in thinking about ethical, legal, and social issues (ELSI) that may arise in the planning and implementation. The content, developed and maintained by the NBSTRN Bioethics and Legal Workgroup, was created to address common topic areas in ELSI of interest to the NBSTRN community groups: researchers, healthcare professionals, families, and advocacy groups, and state NBS programs. ELSI Advantage is organized into different tools and resources, including ELSI 101, which summarizes topics related to ELSI in NBS research. The content addresses common topic areas in ELSI of interest to the NBSTRN community groups: researchers, healthcare professionals, families and advocacy groups, and state NBS programs. Ask ELSA! is an interactive avatar where you can ask a question about ELSI and NBS research. This online resource draws from a database of NBS Research ELSI topics and addresses questions related to the Common Rule, Informed Consent, and Institutional Review Boards (IRB).

For over sixty years, newborn screening has enabled the early identification and treatment of a variety of genetic diseases - both rare and common. Today molecularly designed therapies are revolutionizing treatment and incrementally, and in some cases, dramatically improving health outcomes in conditions like Cystic Fibrosis (C.F.) and Spinal Muscular Atrophy (SMA). In this era of gene-targeted therapies and pre-symptomatic diagnosis, NBS has emerged as a potential mechanism to screen, diagnose and treat potentially all the estimated seven thousand genetic conditions. These rapid advancements in genomic technologies and treatments present even more ways that public health teams, researchers, clinicians, and families can work together to save the lives of infants with a genetic condition.

The authors can be contacted at abrower@acmg.net

Copyright (c) 2021 Delaware Academy of Medicine / Delaware Public Health Association.

This is an Open Access article distributed under the terms of the Creative Commons Attribution Non-Commercial License (https://creativecommons.org/licenses/by-nc-nd/4.0/) which permits unrestricted non-commercial use, distribution, and reproduction in any medium, provided the original work is properly cited. 